\title{
AN ELECTRICAL COLONY COUNTER
}

\author{
BY \\ W. D. LINSELL \\ From the Department of Bacteriology, St. Mary's Hospital Medical School, London
}

(RECEIVED FOR PUBlication AUgust 29, 1952)

During the studies on the effects of antibiotics on bacterial populations which involved the counting of large numbers of colonies by surface viable count technique, it was apparent that some mechanical device for the actual counting would be of great assistance. Such apparatus would have to be simple to use, capable of recording rapidly, and would have to mark in some way each colony as it was counted to ensure accuracy and avoid counting colonies more than once.

To meet these requirements an electrical system was devised whereby touching a surface colony with a fine straight wire pointer completed an electrical circuit and caused an electrical counting mechanism to register the single contact. The simplest and most compact electrical counter for this purpose was found to be that used by the G.P.O. as a telephone counter. This is a simple solenoid mechanism enclosed in a box measuring in all $9.5 \times 2.5 \times 2.5 \mathrm{~cm}$. which, by a ratchet and pinion, rotated a geared four-number series. This series cannot be set at zero, and so subtraction is necessary when each count is made ; this was found to be of small inconvenience. The counter is exceedingly sensitive to the lightest contact when a potential of 40 volts is employed. Experiments with nutrient agar plate showed that if the electrodes are at opposite sides of the plate, then the voltage should be increased to 60 for maximum sensitivity of action, provided that the plate is not excessively dry.

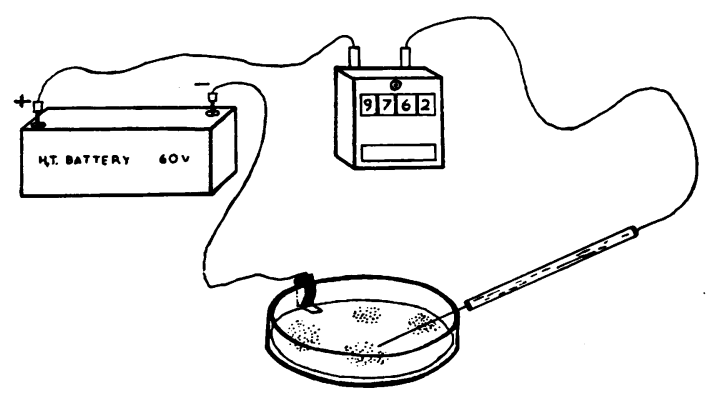

FIG. 1.-A diagram showing the apparatus and electrical circuit.
The diagram (Fig. 1) shows the construction of the apparatus in its simplest form. Current is supplied by a wireless high-tension battery; this in daily use should last for at least a year, and it is more mobile than a mains transformer-rectifier, which can be used in its place equally efficiently. One battery terminal is connected by thin insulated wire to the plate electrode, which consists of small square copper sheet, $5 \times 5 \mathrm{~mm}$., lying on the surface of the agar; one edge of this is turned upwards and soldered to a brass spring clip which grips the side of the plate and to which the wire is attached. With the exception of the copper foot the electrode is insulated. The spring clip is forced down the side of the dish until the copper foot makes firm contact with the agar surface. The pointer contact is an ordinary bacteriological wire holder with a fine straight platinum wire and an insulated handle ; a thin wire passes from the end of the handle to one terminal on the telephone counter; the circuit is completed by a wire from the other terminal on the counter to the battery.

Various types of electrical colony counter have been devised. One well-known variety involves counting the colonies by making ink spots over them on the reverse side of the petri dish; this has some disadvantage in that closely grouped

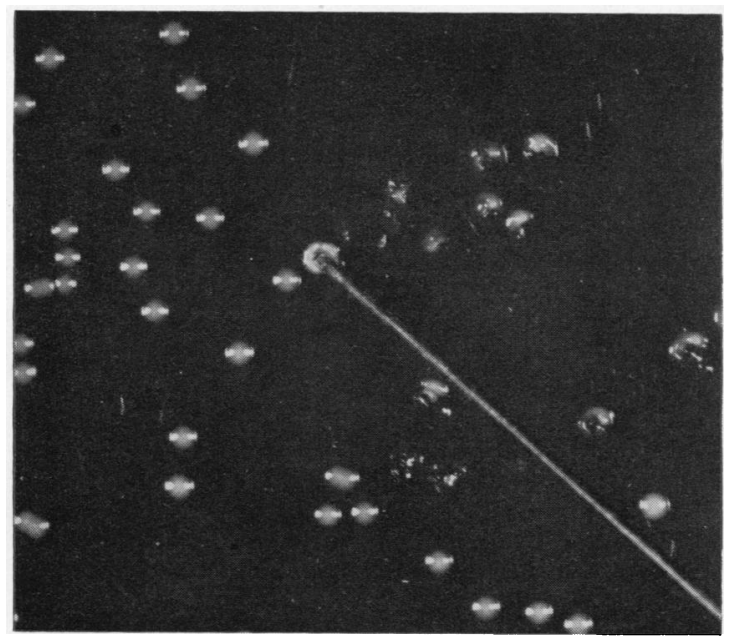

Fig. 2.-Enterococci being counted $(\times 5)$. 
small colonies are difficult to see through medium and glass, especially if the latter is scratched. Counters involving an electrical counting mechanism independent of the actual touching of the colony are more exacting in their manipulation, and counting is consequently slower and more fatiguing.

The apparatus described here can be used for very small colonies under a plate microscope ; Fig. 2 shows colonies of enterococci being counted, and also the very definite way in which the colonies are marked off as they are counted. Contact, however light, by the wire pointer with the colony causes its immediate disruption. As skill develops with use of the apparatus it is not difficult to reach a counting speed of 200 to 250 colonies per minute, and conversation can be carried on during the procedure, since the addition is being recorded automatically ; fatigue is minimal.
The wire can be cleaned in the usual way by heating it red hot in a Bunsen flame. The plates electrode may need to be scraped clean with ao knife after prolonged use. The original appa ratus is enclosed in a box with a holder for then petri dish, which can be illuminated if desired there is a window through which the numbers ono the counter can be observed. The basic design" can be readily modified to suit any particular bench space or experimental requirement.

If a mains supply is desired a transforme? reducing to 100 volts should be used in conjunc tion with a rectifier to convert to direct currentos the final potential being about 60 volts. The counter obtained from the G.P.O. costs 24 s. $6 \mathrm{~d}$.

I wish to thank the Chief Engineer, G.P.O. Telephones, for supplying the telephone counter, and the Photographic Department of St. Mary's Hospitab Medical School for the illustrations. 